\title{
Synthesis and antiulcer activity of 2-[5-substituted-1- $H$-benzo(d) imidazol-2-yl sulfinyl]methyl-3-substituted quinazoline-4-(3H) ones
}

\author{
AVINASH PATIL ${ }^{1, *}$, SWASTIKA GANGULY ${ }^{2}$ and SANJAY SURANA ${ }^{3}$ \\ ${ }^{1}$ Smt. S.S. Patil College of Pharmacy, Chopda 425107 \\ ${ }^{2}$ Department of Pharmaceutical Sciences, Birla Institute of Technology, Mesra, Ranchi 835215 \\ ${ }^{3}$ R.C. Patel College of Pharmacy, Shirpur 425405 \\ e-mail: avinashay_princ@rediffmail.com
}

MS received 3 July 2009; accepted 7 September 2009

\begin{abstract}
H-benzo(d)imidazol-2-yl sulfinyl]methyl-3-substituted quinazoline-4$(3 H)$-one derivatives were synthesized and tested for antiulcer activity against pylorus ligation-induced, aspirin induced and ethanol induced ulcer in rat model. All the synthesized compounds were characterized by using IR, MS and ${ }^{1} \mathrm{H}$ NMR spectral and elemental analysis. The compounds were scramed for their antiulcer activity: compounds $\mathbf{5 k}$ and $\mathbf{5 n}$ showed higher activity than omeprazole used as standard.
\end{abstract}

Keywords. Synthesis; 4-quinazolinone; benzimidazole; antiulcer activity.

\section{Introduction}

Benzimidazole sulfinyl methyl pyridine is a wellestablished class of $\mathrm{H}^{+} / \mathrm{K}^{+}$ATPase inhibitors, therapeutically useful in the treatment of acute and chronic ulcer conditions. ${ }^{1}$ In the past few years, research for new antiulcer agents has focused on numerous structural patterns of benzimidazole sulfinyl methyl pyridine moiety by substitution on the benzimidazole ring, methyl sulfinyl chain and pyridine. ${ }^{2}$ Besides this studies, another simple chemical modification was done by an insertion of pyrimidine ring instead of pyridine in the benzimidazole sulfinyl methyl pyridine moity, resulted in increase in antiulcer and antisecretory activity. ${ }^{3}$ In addition, in recent years there has been an increasing interest in the chemistry of $4(3 \mathrm{H})$-quinazolinones because of their biological significance. ${ }^{4,5}$ In view of these reports, it was thought worthwhile to synthesize and investigate the compounds in which the benzimidazole derivatives have been linked with the quinazoline moiety.

On the basis of the above mentioned reports, the present work is concerned with the synthesis of different 2-[5-substituted 1- $H$ benzo(d)imidazol-2-yl sulfinyl]methyl-3-substituted quinazoline-4-(3H)-one derivatives with the objective of discovering novel antiulcer agents.

\footnotetext{
*For correspondence
}

\section{Experimental}

\subsection{Materials, method and instruments}

Melting points were determined with Lab line melting point apparatus and are uncorrected. Infra-Red spectra were recorded on a Shimadzu 8400-s spectrophotometer using $\mathrm{KBr}$ pellets. Proton nuclear magnetic resonance $\left({ }^{1} \mathrm{H}\right.$ NMR) spectra were recorded on a Varion $300 \mathrm{MHz}$ spectrometer using $\mathrm{CDCI}_{3}$, as solvent with TMS as an internal standard. Mass spectral data was recorded on Shimadzu QP5050 spectrophotometer. Elemental analysis $(\mathrm{C}, \mathrm{H}, \mathrm{N})$ were performed on a FLASH EA 1112 analyzer and were within \pm 0.4 of the theoretical value. The reactions were monitored by thin layer chromatography (TLC) using silica gel-G (benzene:ethanol, $1: 5$ ).

\subsection{General method for synthesis of compounds $(5 \mathbf{a}-\mathbf{0})$}

2.2a 2-((1-H Benzo[d]imidazol-2-yl sulfinyl) methyl)-3-(3,4 dimethoxy phenyl) quinazolin 4(3H)one (5a): To a solution of $4 \mathrm{a}(1 \mathrm{~g}, 2 \mathrm{mmol})$ in dichloromethane $(50 \mathrm{~mL})$ hydrogen peroxide $(30 \%$ $\mathrm{w} / \mathrm{v}, 0.2 \mathrm{~mL}, 2 \mathrm{mmol})$ in acetic acid $(5 \mathrm{~mL})$ was added drop-wise in reaction mixture. The mixture was heated at $50-60^{\circ} \mathrm{C}$ under stirring for $8-10 \mathrm{~h}$. Thereafter, the solvent was removed under reduced pressure. Residue was added in ice cold water to 
yield a precipitate which was filtered and recrystallized from ethanol and shows m.p.: $240-242^{\circ} \mathrm{C}$ in $68 \%$ yield.

$R_{\mathrm{f}}=0.79 ; \quad \mathrm{IR}:\left(\mathrm{KBr} / \mathrm{cm}^{-1}\right): 1514 \quad(\mathrm{C}=\mathrm{C}), \quad 1714$ $(\mathrm{C}=\mathrm{O}), 2980(\mathrm{C}-\mathrm{H}$ alkyl), $3126(\mathrm{C}-\mathrm{H}$ Ar $), 1467$ $(\mathrm{C}=\mathrm{N}), 1543(\mathrm{C}-\mathrm{N}), 3153(\mathrm{~N}-\mathrm{H}), 740 \quad(\mathrm{C}-\mathrm{S}-\mathrm{C})$, $1033(\mathrm{~S}=\mathrm{O}) 1261(\mathrm{C}-\mathrm{O}-\mathrm{C})$.

${ }^{1} \mathrm{H}$ NMR $\left(300 \mathrm{MHz}, \mathrm{CDCI}_{3}\right) \quad \delta_{\mathrm{ppm}}: 3.9 \quad(s, 6 \mathrm{H}$, $\left.\mathrm{OCH}_{3}\right), 5.0\left(s, 2 \mathrm{H}, \underline{\mathrm{CH}}_{2} \mathrm{SO}\right), 12.4(s, 1 \mathrm{H}, \underline{\mathrm{NH}}), 6 \cdot 4-$ $8.0(\mathrm{~m}, 11 \mathrm{H}, \mathrm{ArH}) . \mathrm{MS}(\mathrm{m} / \mathrm{z}) 460[\mathrm{M}]^{+}$; Anal. Calcd. for $\mathrm{C}_{24} \mathrm{H}_{20} \mathrm{~N}_{4} \mathrm{O}_{4} \mathrm{~S}_{1}: \mathrm{C}, 62 \cdot 60 ; \mathrm{H}, 4 \cdot 34 ; \mathrm{N}, 12 \cdot 17$. Found: C, 62.80; H, 4.61; N, 12.07. Compounds 5bo were obtained similarly.

$2.2 \mathrm{~b} \quad 2-((1-H \quad B e n z o[d]$ imidazol-2-yl sulfinyl) methyl)-3-(pyridyl-2-yl) quinazolin-4(3H)- one (5b): Yield: $0.64 \mathrm{~g}(62 \%) ;$ m.p.: $252-254^{\circ} \mathrm{C} ; R_{\mathrm{f}}: 0.80$, IR $\left(\mathrm{KBr} / \mathrm{cm}^{-1}\right): 1592(\mathrm{C}=\mathrm{C}), 1680(\mathrm{C}=\mathrm{O}), 2950(\mathrm{C}-\mathrm{H}$ alkyl), $1668(\mathrm{C}=\mathrm{N}), 1514(\mathrm{C}-\mathrm{N}), 3180(\mathrm{~N}-\mathrm{H}), 785$ (C-S-C), $1030(\mathrm{~S}=\mathrm{O})$.

${ }^{1} \mathrm{H}$ NMR $\left(300 \mathrm{MHz}, \mathrm{CDCI}_{3}\right) \delta_{\mathrm{ppm}}: 5 \cdot 1(s, 2 \mathrm{H}$, $\left.\mathrm{CH}_{2} \mathrm{SO}\right), 11.8(s, 1 \mathrm{H}, \underline{\mathrm{NH}}$ benz $), 6.4-8.4(m, 12 \mathrm{H}$, ArH). MS $(m / z) 401 \quad\left[\mathrm{M}^{+}\right.$; Anal. Calcd. for $\mathrm{C}_{21} \mathrm{H}_{15} \mathrm{~N}_{5} \mathrm{O}_{2} \mathrm{~S}_{1}$ : C, 62.84; H, 3.74; N, 17.45. Found: C, $62.92 ; \mathrm{H}, 3.91 ; \mathrm{N}, 17.28$.

2.2c 2-((1-H Benzo[d]imidazol-2-yl sulfinyl) methyl)-3-(pyridyl-3-yl) quinazolin-4(3H)-one (5c): Yield: $0.74 \mathrm{~g}(72 \%) ;$ m.p.: $240-242^{\circ} \mathrm{C}, R_{\mathrm{f}}: 0.81$, IR $\left(\mathrm{KBr} / \mathrm{cm}^{-1}\right): 1585(\mathrm{C}=\mathrm{C}), 1670(\mathrm{C}=\mathrm{O}), 2945(\mathrm{C}-\mathrm{H}$ alkyl), $1660(\mathrm{C}=\mathrm{N}), 1524(\mathrm{C}-\mathrm{N}), 3210(\mathrm{~N}-\mathrm{H}), 782$ $(\mathrm{C}-\mathrm{S}-\mathrm{C}), 1030(\mathrm{~S}=\mathrm{O})$.

${ }^{1} \mathrm{H} \quad \mathrm{NMR}\left(300 \mathrm{MHz}, \mathrm{CDCI}_{3}\right) \quad \delta_{\mathrm{ppm}}: 4.9 \quad(s, 2 \mathrm{H}$, $\left.\mathrm{C}_{2} \mathrm{SO}\right), 11.8(s, 1 \mathrm{H}, \underline{\mathrm{NH}}$ benz $), 6 \cdot 4-8.2(m, 12 \mathrm{H}$, ArH). MS $(\mathrm{m} / \mathrm{z}) 401 \quad[\mathrm{M}]^{+}$; Anal. Calcd. for $\mathrm{C}_{21} \mathrm{H}_{15} \mathrm{~N}_{5} \mathrm{O}_{2} \mathrm{~S}_{1}: \mathrm{C}, 62 \cdot 84 ; \mathrm{H}, 3 \cdot 74 ; \mathrm{N}, 17 \cdot 45$. Found: C, 62.72; H, 4.04; N, 17.38.

$2.2 \mathrm{~d} \quad 2-((1-H \quad$ Benzo[d]imidazol-2-yl sulfinyl) methyl)-3-(pyrazine-2-yl) quinazolin-4(3H)-one (5d): Yield: $0.72 \mathrm{~g}(70 \%)$; m.p.: $292-294^{\circ} \mathrm{C}, R_{\mathrm{f}}: 0.82$, IR $\left(\mathrm{KBr} / \mathrm{cm}^{-1}\right): 1632(\mathrm{C}=\mathrm{C}), 1700(\mathrm{C}=\mathrm{O}), 2940(\mathrm{C}-\mathrm{H}$ alkyl), $1642(\mathrm{C}=\mathrm{N}), 1468(\mathrm{C}-\mathrm{N}), 3250(\mathrm{~N}-\mathrm{H}), 742$ (C-S-C), $1029(\mathrm{~S}=\mathrm{O}) .{ }^{1} \mathrm{H}$ NMR $\left(300 \mathrm{MHz}, \mathrm{CDCI}_{3}\right)$ $\delta_{\text {ppm }}: 4.9\left(s, 2 \mathrm{H}, \mathrm{CH}_{2} \mathrm{SO}\right) ; 11.8(s, 2 \mathrm{H}, \underline{\mathrm{NH}}), 6.4-8.0$ $(\mathrm{m}, 12 \mathrm{H}, \mathrm{ArH})$. MS $(\mathrm{m} / \mathrm{z}) 402[\mathrm{M}]^{+}$; Anal. Calcd. for $\mathrm{C}_{20} \mathrm{H}_{14} \mathrm{~N}_{6} \mathrm{O}_{2} \mathrm{~S}_{1}$ : C, 59.70; H, 3.48; N, 20.89. Found: C, 59.92; H, 3.78; N, 21.20.

2.2e 2-((1-H Benzo[d]imidazol-2-yl sulfinyl) methyl)3-(tetrazole-5-yl) quinazolin-4(3H)-one (5e): Yield: $056 \mathrm{~g}(54 \%)$; m.p.: above $300^{\circ} \mathrm{C} ; R_{\mathrm{f}}: 0.70$, IR
$\left(\mathrm{KBr} / \mathrm{cm}^{-1}\right): 1635(\mathrm{C}=\mathrm{C}), 1678(\mathrm{C}=\mathrm{O}), 2920(\mathrm{C}-\mathrm{H}$ alkyl), $1642(\mathrm{C}=\mathrm{N}), 1410(\mathrm{C}-\mathrm{N}), 3320(\mathrm{~N}-\mathrm{H}), 716$ $(\mathrm{C}-\mathrm{S}-\mathrm{C}), 1021(\mathrm{~S}=\mathrm{O}), 1480(\mathrm{~N}=\mathrm{N})$.

${ }^{1} \mathrm{H}$ NMR $\left(300 \mathrm{MHz}, \mathrm{CDCI}_{3}\right) \delta_{\mathrm{ppm}}: 12 \cdot 2(s, 1 \mathrm{H}, \underline{\mathrm{NH}}$ benz), $12.5(s, 1 \mathrm{H}, \underline{\mathrm{NH}}$ tetrazole $), 5.3(s, 2 \mathrm{H}$, $\left.\mathrm{C}_{2} \mathrm{SO}\right), 6 \cdot 9-8.5(\mathrm{~m}, 8 \mathrm{H}, \mathrm{ArH}) . \mathrm{MS}(\mathrm{m} / \mathrm{z})$ 392[M] $]^{+}$; Anal. Calcd. for $\mathrm{C}_{17} \mathrm{H}_{12} \mathrm{~N}_{8} \mathrm{O}_{2} \mathrm{~S}_{1}$ : C, 52.04; H, 3.06; $\mathrm{N}, 28 \cdot 57$. Found: C, 52.32; H, 3.31; N, 28.68.

$2.2 \mathrm{f} \quad 2-((5-M e t h o x y$ benzo[d]imidazol-2-ylsulfinyl) methyl)-3-(3,4 dimethoxy phenyl) quinazolin-4(3H)one (5f): Yield: 0.66g (64\%); m.p.: $236-238^{\circ} \mathrm{C}$; $R_{\mathrm{f}}: 0 \cdot 78$, IR $\left(\mathrm{KBr} / \mathrm{cm}^{-1}\right): 1521(\mathrm{C}=\mathrm{C}), 1710(\mathrm{C}=\mathrm{O})$, $2950(\mathrm{C}-\mathrm{H}$ alkyl), $1610(\mathrm{C}=\mathrm{N}), 1467(\mathrm{C}-\mathrm{N}), 3190$ $(\mathrm{N}-\mathrm{H}), 780$ (C-S-C), 1265 (C-O-C), 1049 (S=O).

${ }^{1} \mathrm{H}$ NMR $\left(300 \mathrm{MHz}, \mathrm{CDCI}_{3}\right) \delta_{\mathrm{ppm}}: 3.8(s, 9 \mathrm{H}$, $\left.\mathrm{OCH}_{3}\right), 12 \cdot 0(s, 1 \mathrm{H}, \underline{\mathrm{NH}}$ benz $), 4 \cdot 8\left(s, 2 \mathrm{H}, \underline{\mathrm{CH}_{2} \mathrm{SO}}\right)$, 6.6-7.8 ( $m, 8 \mathrm{H}, \operatorname{ArH})$. MS $(\mathrm{m} / \mathrm{z}) 491[\mathrm{M}]^{+}$; Anal. Calcd. for $\mathrm{C}_{25} \mathrm{H}_{22} \mathrm{~N}_{4} \mathrm{O}_{5} \mathrm{~S}_{1}: \mathrm{C}, 61.09 ; \mathrm{H}, 4.48 ; \mathrm{N}$, 11.40. Found: C, 61.32; H, 4.51; N, 11.30.

$2.2 \mathrm{~g}$ 2-((5-Methoxy benzo[d]imidazol-2-yl sulfinyl) methyl)-3-(pyridyl-2-yl) quinazolin-4(3H)-one (5g): Yield: $0.69 \mathrm{~g}(67 \%) ;$ m.p.: $260-261^{\circ} \mathrm{C}, R_{\mathrm{f}}: 0.78$, IR $\left(\mathrm{KBr} / \mathrm{cm}^{-1}\right): 1570(\mathrm{C}=\mathrm{C}), 1692(\mathrm{C}=\mathrm{O}), 2940(\mathrm{C}-\mathrm{H}$ alkyl), $1668(\mathrm{C}=\mathrm{N}), 1514(\mathrm{C}-\mathrm{N}), 3200(\mathrm{~N}-\mathrm{H}), 780$ (C-S-C), $1265(\mathrm{C}-\mathrm{O}-\mathrm{C}), 1033(\mathrm{~S}=\mathrm{O})$.

${ }^{1} \mathrm{H}$ NMR $\left(300 \mathrm{MHz}, \mathrm{CDCI}_{3}\right) \delta_{\mathrm{ppm}}: 3.9(s, 3 \mathrm{H}$, $\left.\mathrm{OCH}_{3}\right), 11.9(s, 1 \mathrm{H}, \underline{\mathrm{NH}}$ benz $), 5.0\left(\mathrm{~s}, 2 \mathrm{H}, \underline{\mathrm{CH}_{2}} \mathrm{SO}\right)$, 6.5-8.5 ( $m, 8 \mathrm{H}, \operatorname{ArH})$. MS $(\mathrm{m} / \mathrm{z}) 431[\mathrm{M}]^{+}$; Anal. Calcd. for $\mathrm{C}_{22} \mathrm{H}_{17} \mathrm{~N}_{5} \mathrm{O}_{3} \mathrm{~S}_{1}: \mathrm{C}, 61.25 ; \mathrm{H}, 3.94 ; \mathrm{N}$, 16.24. Found: C, 61.52; H, 4.21; N, 16.38.

$2.2 \mathrm{~h} \quad 2-((5-M e t h o x y$ benzo[d]imidazol-2-yl sulfinyl) methyl)-3-(pyridyl-3-yl) quinazolin-4(3H)-one (5h): Yield: $0.66 \mathrm{~g}(65 \%) ;$ m.p.: $228-230^{\circ} \mathrm{C}, R_{\mathrm{f}}: 0.82$, IR $\left(\mathrm{KBr} / \mathrm{cm}^{-1}\right): 1580(\mathrm{C}=\mathrm{C}), 1670(\mathrm{C}=\mathrm{O}), 2952(\mathrm{C}-\mathrm{H}$ alkyl), $1660(\mathrm{C}=\mathrm{N}), 1524(\mathrm{C}-\mathrm{N}), 3210(\mathrm{~N}-\mathrm{H}), 782$ $(\mathrm{C}-\mathrm{S}-\mathrm{C}), 1270(\mathrm{C}-\mathrm{O}-\mathrm{C}), 1033(\mathrm{~S}=\mathrm{O})$.

${ }^{1} \mathrm{H}$ NMR $\left(300 \mathrm{MHz}, \mathrm{CDCI}_{3}\right) \quad \delta_{\mathrm{ppm}}: 5.0(s, 2 \mathrm{H}$, $\left.\mathrm{C}_{2} \mathrm{SO}\right), 3.9\left(s, 3 \mathrm{H}, \mathrm{OC}_{3}\right), 12.3(s, 1 \mathrm{H}, \underline{\mathrm{NH}}$ benz), 6.6-7.4 (m, 8H, ArH). MS $(m / z) 431\left[\mathrm{M}^{+}\right.$; Anal. Calcd. for $\mathrm{C}_{22} \mathrm{H}_{17} \mathrm{~N}_{5} \mathrm{O}_{3} \mathrm{~S}_{1}: \mathrm{C}, 61.25 ; \mathrm{H}, 3.94 ; \mathrm{N}$, 16.24. Found: C, 61.52; H, 4.31; N, 16-38.

$2.2 \mathrm{i}$ 2-((5-Methoxy benzo[d]imidazol-2-yl sulfinyl) methyl)-3-(pyrazine-2-yl) quinazolin-4(3H)-one (5i): Yield: $0.74 \mathrm{~g}(72 \%)$; m.p.: $286-288^{\circ} \mathrm{C}, R_{\mathrm{f}}: 0 \cdot 84$, IR $\left(\mathrm{KBr} / \mathrm{cm}^{-1}\right): 1644(\mathrm{C}=\mathrm{C}), 1705(\mathrm{C}=\mathrm{O}), 2932(\mathrm{C}-\mathrm{H}$ alkyl), $1652(\mathrm{C}=\mathrm{N}), 1468(\mathrm{C}-\mathrm{N}), 3240(\mathrm{~N}-\mathrm{H}), 742$ $(\mathrm{C}-\mathrm{S}-\mathrm{C}), 1042(\mathrm{~S}=\mathrm{O}), 1272(\mathrm{C}-\mathrm{O}-\mathrm{C})$. 
${ }^{1} \mathrm{H}$ NMR $\left(300 \mathrm{MHz}, \mathrm{CDCI}_{3}\right) \delta_{\mathrm{ppm}}: 5.0(s, 2 \mathrm{H}$, $\left.\mathrm{CH}_{2} \mathrm{SO}\right), 3.9\left(s, 3 \mathrm{H}, \mathrm{OCH}_{3}\right), 11.8(s, 1 \mathrm{H}, \underline{\mathrm{NH}}), 6 \cdot 6-$ $7.9(\mathrm{~m}, 8 \mathrm{H}, \mathrm{ArH}) . \mathrm{MS}(\mathrm{m} / \mathrm{z}) 432[\mathrm{M}]^{+}$; Anal. Calcd. for $\mathrm{C}_{21} \mathrm{H}_{16} \mathrm{~N}_{6} \mathrm{O}_{3} \mathrm{~S}_{1}$ : C, 58.33; H, 3.70; N, 19.44. Found: C, 58.52; H, 3.90; N,19.22.

$2.2 \mathrm{j} \quad 2-((5-M e t h o x y$ benzo[d]imidazol-2-yl sulfinyl) methyl)-3-(tetrazole-5-yl) quinazolin-4(3H)-one (5j): Yield: $0.61 \mathrm{~g}(59 \%)$; m.p.:above $300^{\circ} \mathrm{C}, R_{\mathrm{f}}: 0.78$, IR $\left(\mathrm{KBr} / \mathrm{cm}^{-1}\right): 1622(\mathrm{C}=\mathrm{C}), 1680(\mathrm{C}=\mathrm{O}), 2910(\mathrm{C}-\mathrm{H}$ alkyl), $1660(\mathrm{C}=\mathrm{N}), 1422(\mathrm{C}-\mathrm{N}), 3420(\mathrm{~N}-\mathrm{H}), 718$ $(\mathrm{C}-\mathrm{S}-\mathrm{C}), 1268(\mathrm{C}-\mathrm{O}-\mathrm{C}), 1069(\mathrm{~S}=\mathrm{O}) 1482(\mathrm{~N}=\mathrm{N})$.

${ }^{1} \mathrm{H}$ NMR $\left(300 \mathrm{MHz}, \mathrm{CDCI}_{3}\right) \delta_{\mathrm{ppm}}: 5 \cdot 0(s, 2 \mathrm{H}$, $\left.\mathrm{C}_{2} 2 \mathrm{SO}\right), 3.8\left(s, 3 \mathrm{H}, \mathrm{OC}_{1}\right), 11.9(s, 1 \mathrm{H}, \underline{\mathrm{NH}}$ benz.), $12 \cdot 4(s, 1 \mathrm{H}, \underline{\mathrm{NH}}$ tetrazole $), 6 \cdot 6-8 \cdot 2(m, 8 \mathrm{H}, \mathrm{ArH})$. MS $(\mathrm{m} / \mathrm{z}) 422[\mathrm{M}]^{+}$; Anal. Calcd. for $\mathrm{C}_{18} \mathrm{H}_{14} \mathrm{~N}_{8} \mathrm{O}_{3} \mathrm{~S}_{1}$ : C, 51.18; H, 3.31; N, 26.54. Found: C, 41.52; H, $3 \cdot 41 ; \mathrm{N}, 26 \cdot 38$.

$2.2 \mathrm{k}$ 2-((5-Difluromethoxy benzo[d]imidazol-2-yl sulfinyl) methyl)-3-(3,4 dimethoxy phenyl) quinazolin4(3H)-one (5k): Yield: 0.66 g (64\%); m.p.: 222 $224^{\circ} \mathrm{C}, R_{\mathrm{f}}: 0 \cdot 76, \mathrm{IR}\left(\mathrm{KBr} / \mathrm{cm}^{-1}\right): 1519(\mathrm{C}=\mathrm{C}), 1757$ $(\mathrm{C}=\mathrm{O}), 2930(\mathrm{C}-\mathrm{H}$ alkyl), $1467(\mathrm{C}=\mathrm{N}), 1537(\mathrm{C}-\mathrm{N})$, $3110(\mathrm{~N}-\mathrm{H}), 802$ (C-S-C), $1280(\mathrm{C}-\mathrm{O}-\mathrm{C}), 1031$ $(\mathrm{S}=\mathrm{O})$.

${ }^{1} \mathrm{H}$ NMR $\left(300 \mathrm{MHz}, \mathrm{CDCI}_{3}\right) \delta_{\mathrm{ppm}}: 3.9(s, 6 \mathrm{H}$, $\left.\mathrm{OCH}_{3}\right), 12 \cdot 6(s, 1 \mathrm{H}, \underline{\mathrm{NH}}$ benz $), 5 \cdot 0\left(s, 2 \mathrm{H}, \mathrm{CH}_{2} \mathrm{SO}\right)$, $6 \cdot 6\left(t, 1 \mathrm{H}, \mathrm{OCHF}_{2}\right), 6 \cdot 8-7 \cdot 6(\mathrm{~m}, 8 \mathrm{H}, \mathrm{ArH}) . \mathrm{MS}(\mathrm{m} / \mathrm{z})$ $526[\mathrm{M}]^{+}$; Anal. Calcd. for $\mathrm{C}_{25} \mathrm{H}_{20} \mathrm{~N}_{4} \mathrm{O}_{5} \mathrm{~S}_{1} \mathrm{~F}_{2}: \mathrm{C}$, $57.03 ; \mathrm{H}, 3.80 ; \mathrm{N}, 10 \cdot 64$. Found: C, 57.32; H, 3.91; $\mathrm{N}, 10 \cdot 78$.

2.21 2-((5-Difluromethoxy benzo[d]imidazol-2-yl sulfinyl) methyl)-3-(pyridyl-2-yl) quinazolin-4(3H)one (5l): Yield: $0.66 \mathrm{~g}(64 \%)$; m.p.:232-234 ${ }^{\circ} \mathrm{C}, R_{\mathrm{f}}$ : $0.78 \mathrm{IR}\left(\mathrm{KBr} / \mathrm{cm}^{-1}\right): 1585(\mathrm{C}=\mathrm{C}), 1692(\mathrm{C}=\mathrm{O}), 2932$ (C-H alkyl), $1668(\mathrm{C}=\mathrm{N}), 1516(\mathrm{C}-\mathrm{N}), 3190(\mathrm{~N}-\mathrm{H})$, 780 (C-S-C), 1265 (C-O-C), $1010(\mathrm{~S}=\mathrm{O})$.

${ }^{1} \mathrm{H}$ NMR $\left(300 \mathrm{MHz}, \mathrm{CDCI}_{3}\right) \delta_{\mathrm{ppm}}: 5 \cdot 1(s, 2 \mathrm{H}$, $\left.\mathrm{CH}_{2} \mathrm{SO}\right), 6.5\left(t, 1 \mathrm{H}, \mathrm{OCHF}_{2}\right), 12.0(s, 1 \mathrm{H}, \underline{\mathrm{NH}}$ benz.), 6.8-8.2 ( $m, 8 \mathrm{H}, \mathrm{ArH})$. MS $(\mathrm{m} / \mathrm{z}) 467[\mathrm{M}]^{+}$; Anal. Calcd. for $\mathrm{C}_{22} \mathrm{H}_{15} \mathrm{~N}_{5} \mathrm{O}_{3} \mathrm{~S}_{1} \mathrm{~F}_{2}: \mathrm{C}, 56 \cdot 53 ; \mathrm{H}, 3.21$; N, 14.98. Found: C, 56.72; H, 3.41; N, 15.28.

$2.2 \mathrm{~m} \quad$ 2-((5-Difluromethoxy benzo[d]imidazol-2-yl sulfinyl) methyl)-3-(pyriyl-3-yl) quinazolin-4(3H)one $(\mathbf{5 m})$ : Yield: $0.70 \mathrm{~g}(68 \%)$; m.p.: $208-210^{\circ} \mathrm{C}$, $R_{\mathrm{f}}: 0 \cdot 84, \mathrm{IR}\left(\mathrm{KBr} / \mathrm{cm}^{-1}\right): 1588(\mathrm{C}=\mathrm{C}), 1670(\mathrm{C}=\mathrm{O})$, $2910(\mathrm{C}-\mathrm{H}$ alkyl), $1662(\mathrm{C}=\mathrm{N}), 1525(\mathrm{C}-\mathrm{N}), 3260$ (N-H), 785 (C-S-C), 1272 (C-O-C), 1031 (S=O).
${ }^{1} \mathrm{H}$ NMR $\left(300 \mathrm{MHz}, \mathrm{CDCI}_{3}\right) \delta_{\mathrm{ppm}}: 5 \cdot 1(s, 2 \mathrm{H}$, $\left.\mathrm{C}_{2} \mathrm{SO}\right), 6 \cdot 4\left(t, 1 \mathrm{H}, \quad \mathrm{OCHF}_{2}\right), 12 \cdot 2(s, 1 \mathrm{H}, \underline{\mathrm{NH}}$ benz.), 6.4-8.4 ( $m, 8 \mathrm{H}, \mathrm{ArH})$. MS $(\mathrm{m} / \mathrm{z}) 467[\mathrm{M}]^{+}$; Anal. Calcd for $\mathrm{C}_{22} \mathrm{H}_{15} \mathrm{~N}_{5} \mathrm{O}_{3} \mathrm{~S}_{1} \mathrm{~F}_{2}$ : C, 56.53; H, 3.21; $\mathrm{N}, 14.98$. Found: C, 56.62; H, 3.41; N, 14.78.

2.2n 2-((5-Difluromethoxy benzo[d]imidazol-2-yl sulfinyl) methyl)-3(pyrazine-2-yl) quinazolin-4(3H)one (5n): Yield: $0.72 \mathrm{~g}(70 \%)$; m.p.: $272-274^{\circ} \mathrm{C}$, $R_{\mathrm{f}}: 0 \cdot 85, \mathrm{IR}\left(\mathrm{KBr} / \mathrm{cm}^{-1}\right): 1641(\mathrm{C}=\mathrm{C}), 1705(\mathrm{C}=\mathrm{O})$, 2932 (C-H alkyl), $1655(\mathrm{C}=\mathrm{N}), 1468(\mathrm{C}-\mathrm{N}), 3232$ $(\mathrm{N}-\mathrm{H}), 742(\mathrm{C}-\mathrm{S}-\mathrm{C}), 1039(\mathrm{~S}=\mathrm{O}), 1272(\mathrm{C}-\mathrm{O}-\mathrm{C})$.

${ }^{1} \mathrm{H}$ NMR $\left(300 \mathrm{MHz}, \mathrm{CDCI}_{3}\right) \delta_{\mathrm{ppm}}: 5.0(s, 2 \mathrm{H}$, $\left.\mathrm{C}_{2} \mathrm{SO}\right), 12 \cdot 1(s, 2 \mathrm{H}, \underline{\mathrm{NH}}), 6 \cdot 6\left(t, 1 \mathrm{H}, \mathrm{OCHF}_{2}\right), 6 \cdot 8-$ $8.2(\mathrm{~m}, 8 \mathrm{H}, \mathrm{ArH}) . \mathrm{MS}(\mathrm{m} / \mathrm{z}) 468[\mathrm{M}]^{+}$; Anal. Calcd. for $\mathrm{C}_{21} \mathrm{H}_{14} \mathrm{~N}_{6} \mathrm{O}_{3} \mathrm{~S}_{1} \mathrm{~F}_{2}$ : C, 53.84; $\mathrm{H}, 2.99 ; \mathrm{N}, 17.94$. Found: C, 53.94; H, 3.21; N, 17.98.

2.20 2-((5-Difluromethoxybenzo[d]imidazol-2-ylsulfinyl) methyl)-3-(tetrazole-5-yl) quinazolin4(3H)-one (5o): Yield: 0.56 g (55\%); m.p.: 297$298^{\circ} \mathrm{C}, R_{\mathrm{f}}: 0.70, \mathrm{IR}\left(\mathrm{KBr} / \mathrm{cm}^{-1}\right): 1506(\mathrm{C}=\mathrm{C}), 1695$ $(\mathrm{C}=\mathrm{O}), 2950(\mathrm{C}-\mathrm{H}$ alkyl $), 1660(\mathrm{C}=\mathrm{N}), 1460(\mathrm{C}-\mathrm{N})$, $3450(\mathrm{~N}-\mathrm{H}), 740(\mathrm{C}-\mathrm{S}-\mathrm{C}), 1284(\mathrm{C}-\mathrm{O}-\mathrm{C}), 1041$ $(\mathrm{S}=\mathrm{O}) 1470(\mathrm{~N}=\mathrm{N})$.

${ }^{1} \mathrm{H}$ NMR $\left(300 \mathrm{MHz}, \mathrm{CDCI}_{3}\right) \delta_{\mathrm{ppm}}: 12 \cdot 4(s, 1 \mathrm{H}, \underline{\mathrm{NH}}$ tetrazole $), 12 \cdot 0(s, 1 \mathrm{H}, \underline{\mathrm{NH}}$ benz $), 5 \cdot 1 \quad(s, 2 \mathrm{H}$, $\left.\mathrm{CH}_{2} \mathrm{SO}\right), 6.8\left(t, 1 \mathrm{H}, \mathrm{OCHF}_{2}\right), 7 \cdot 0-8.6(m, 8 \mathrm{H}, \mathrm{ArH})$. MS $(m / z) 458[\mathrm{M}]^{+}$; Anal. Calcd. for $\mathrm{C}_{18} \mathrm{H}_{12} \mathrm{~N}_{8} \mathrm{O}_{3} \mathrm{~S}_{1} \mathrm{~F}_{2}$. C, 47.16; H, 2.62; N, 24.45. Found: C, 47.42; H, $2 \cdot 81 ; \mathrm{N}, 24 \cdot 38$.

\subsection{Antiulcer screening}

2.3a Pylorus ligation-induced gastric ulcer: The different doses were screened for antiulcer activity by pylorus ligation in shay rat method using Omeprazole as standard. ${ }^{6}$ Wistar Albino rats of either sex (150-200 g) were kept in the departmental animal house at room temperature $25-30^{\circ} \mathrm{C}$. Rats were divided into various groups. Omeprazole (10 and $20 \mathrm{mg} / \mathrm{kg})$ and pure compounds 5a-o (10 and $20 \mathrm{mg} / \mathrm{kg}$ ), were suspended in $1 \%$ suspension of CMC (Carboxy methyl cellulose) in distilled water and administered by oral route. The animals were fasted for $48 \mathrm{~h}$ prior to the experiment, but had free access to water. After the fasting period, the animals were given the drug samples p.o, $1 \mathrm{~h}$ prior to the ligation. Thereafter, the rats were anaesthetized with anesthetic ether. An incision of $1 \mathrm{~cm}$ length in the abdomen just below the sternum was made. The 


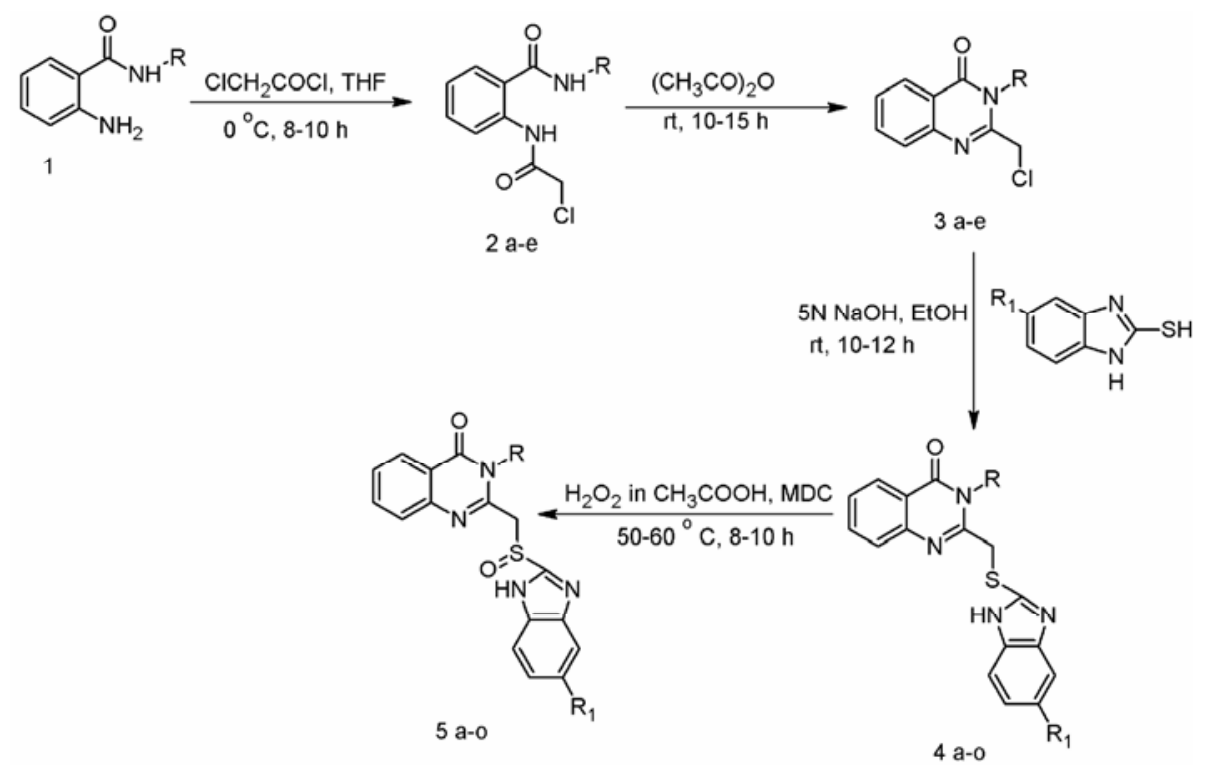

Scheme 1. Reagents and conditions: (i) $\mathrm{ClCH}_{2} \mathrm{COCl}, \mathrm{THF}, 0^{\circ} \mathrm{C}, 8-10 \mathrm{~h}$; (ii) acetic anhydride, rt, $10-15 \mathrm{~h}$; (iii) $5 \mathrm{~N} \mathrm{NaOH}$, EtOH, rt, 10-12 h; (iv) $\mathrm{H}_{2} \mathrm{O}_{2}$ in acetic acid, $\mathrm{MDC}, 50-60^{\circ} \mathrm{C}, 8-10 \mathrm{~h}$.

stomach was exposed. A thread was passed around the pyloric sphincter and a light knot was applied to it taking due care that no blood vessel was tied along the knot. Then incision was closed by stitching the abdominal wall by a thread. The underlying skin was cleaned of any bleeding. An antiseptic cream was applied over the wound. Thereafter, the animal was kept in a separate cage and allowed to recover. Four hours later these animals were sacrificed and the stomach of each of the animals was isolated and cut open through its greater curvature. Its contents emptied into graduated test tube, volume was recorded. $\mathrm{pH}$ was measured by $\mathrm{pH}$ meter. Ulcer score were observed and ulcer index were calculated by One Way ANOVA analysis and results were compared with control by student's t test.

2.3b Aspirin induced gastric ulcer: Wistar Albino rats weighing between (150-200 g) were divided into five animals in group. ${ }^{6}$ The animals are fasted for $24 \mathrm{~h}$. The test drugs were administered orally in $1 \%$ CMC solution $30 \mathrm{~min}$ prior to aspirin at dosage of $200 \mathrm{mg} / \mathrm{kg}$. Four hours later the rats are sacrificed by using anaesthetic ether and their stomachs dissected for the determination of gastric lesions. Ulcer score were observed and ulcer index were calculated by One Way ANOVA analysis and results were compared with control by student's $t$ test.

2.3c Ethanol-induced gastric ulcer: Male Albino wistar rats weighing between 150 and $200 \mathrm{~g}$ were divided into five animals in group. ${ }^{6}$ The animals were fasted for $24 \mathrm{~h}$ with free access to water. Animals were given $10 \mathrm{mg}$ and $20 \mathrm{mg} / \mathrm{kg}$ test compounds and Omeprazole $10 \mathrm{mg}, 20 \mathrm{mg} / \mathrm{kg}$ orally in $1 \%$ CMC solution. $1 \mathrm{~h}$ later, $1 \mathrm{~mL} / 200 \mathrm{~g}$ of $99.00 \%$ alcohol was administered p.o. (per oral - drug doses given to animals orally) to each animal. Animals were sacrificed $1 \mathrm{~h}$ after alcohol administration, stomachs were isolated and cut open along the greater curvature and pinned on a soft board. The gastric mucosa was examined for ulcers. Ulcer scores were observed and ulcer index were calculated by One Way ANOVA analysis and results were compared with control by student's $t$ test.

\subsection{Acute toxicity $\left(L D_{50}\right)$}

Healthy adult Albino mice (20-25 g) of either sex, starved overnight were subjected to acute toxicity studies as per guidelines (AOT No. 425) suggested by Organization for Economic Co-operation and Development (OECD) 2001. The mice were observed continuously for $2 \mathrm{~h}$ for behavioural, neurological and autonomic profiles for any lethality or death for next $48 \mathrm{~h}$.

\section{Results and discussion}

$3.12-((5-S u b s t i t u t e d$ benzo[d]imidazol-2-yl sulfinyl) methyl)-3-substituted quinazolin-4-(3H)-one (5a-o): 


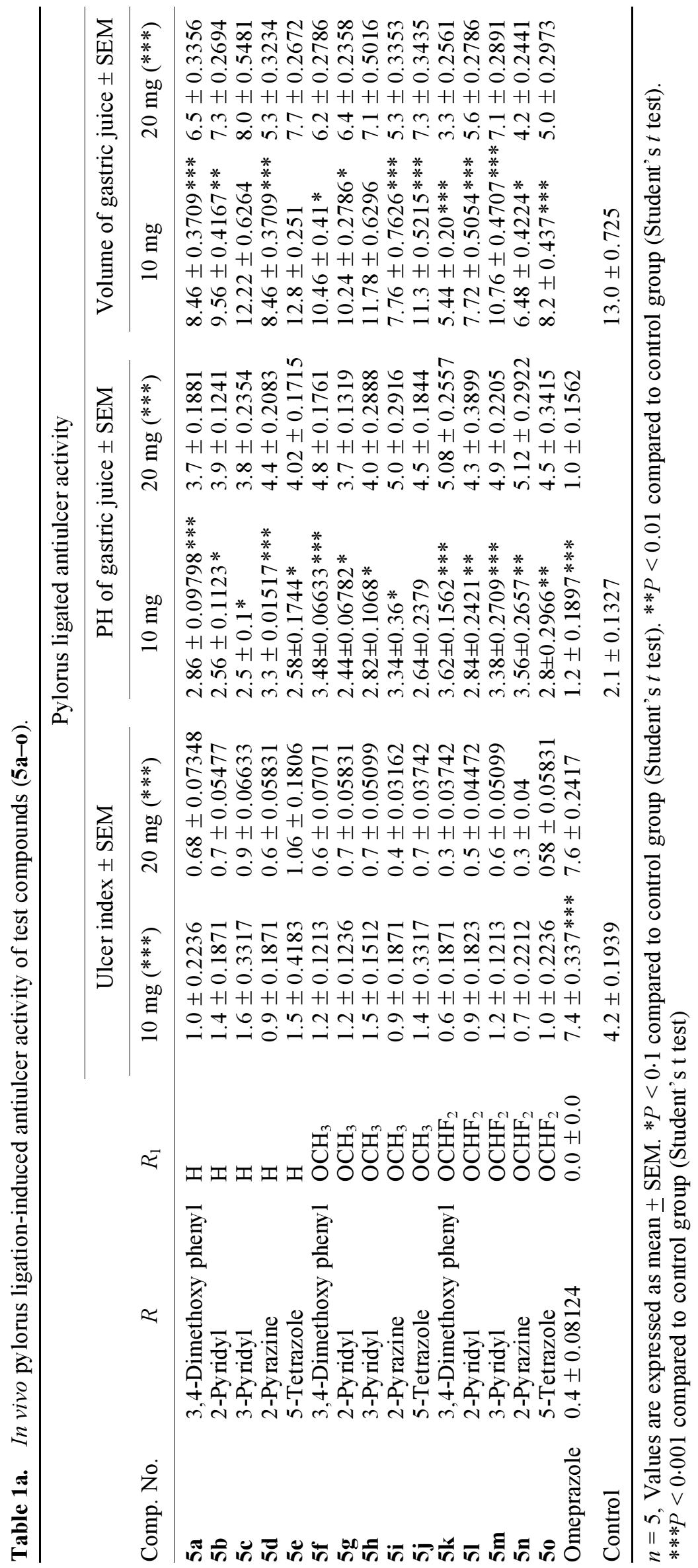


Table 1b. In vivo aspirin induced antiulcer activity of some test compounds.

\begin{tabular}{|c|c|c|c|c|}
\hline \multirow[b]{2}{*}{ Compound no. } & \multirow[b]{2}{*}{$R$} & \multirow[b]{2}{*}{$R_{1}$} & \multicolumn{2}{|c|}{ Ulcer index \pm SEM } \\
\hline & & & $10 \mathrm{mg}(* * *)$ & $20 \mathrm{mg}(* * *)$ \\
\hline $5 \mathbf{a}$ & 3,4-Dimethoxy phenyl & $\mathrm{H}$ & $0 \cdot 6 \pm 0 \cdot 1$ & $0 \cdot 4 \pm 0 \cdot 1$ \\
\hline $5 d$ & 2-Pyrazine & $\mathrm{H}$ & $0.7 \pm 0.1225$ & $0.3 \pm 0.1225$ \\
\hline $5 \mathbf{i}$ & 2-Pyrazine & $\mathrm{OCH}_{3}$ & $0 \cdot 5 \pm 0 \cdot 1581$ & $0.2 \pm 0 \cdot 1225$ \\
\hline $5 \mathbf{k}$ & 3,4-Dimethoxy phenyl & $\mathrm{OCHF}_{2}$ & $0.4 \pm 0.1871$ & $0 \cdot 2 \pm 0 \cdot 1225$ \\
\hline 51 & 2-Pyridyl & $\mathrm{OCHF}_{2}$ & $0 \cdot 4 \pm 0 \cdot 1871$ & $0 \cdot 5 \pm 0 \cdot 0$ \\
\hline $5 n$ & 2-Pyrazine & $\mathrm{OCHF}_{2}$ & $0 \cdot 5 \pm 0 \cdot 1581$ & $0 \cdot 1 \pm 0 \cdot 1$ \\
\hline 50 & 5-Tetrazole & $\mathrm{OCHF}_{2}$ & $0 \cdot 8 \pm 0 \cdot 2$ & $0 \cdot 1 \pm 0 \cdot 1$ \\
\hline Omeprazole & & & $0.5 \pm 0.1581$ & $0 \cdot 0 \pm 0 \cdot 0$ \\
\hline Control & & & & $3 \cdot 8 \pm 0 \cdot 1225$ \\
\hline
\end{tabular}

$n=5$, Values are expressed as mean \pm SEM. $* P<0 \cdot 1$ compared to control group (Student's $t$ test). ${ }^{* * P} P<0.01$ compared to control group (Student's $t$ test). ${ }^{* * *} P<0.001$ compared to control group (Student's $t$ test).

Table 1c. In vivo ethanol induced antiulcer activity of some test compounds.

\begin{tabular}{|c|c|c|c|c|}
\hline \multirow[b]{2}{*}{ Compound no. } & \multirow[b]{2}{*}{$R$} & \multirow[b]{2}{*}{$R_{1}$} & \multicolumn{2}{|c|}{ Ulcer index \pm SEM } \\
\hline & & & $10 \mathrm{mg}(* * *)$ & $20 \mathrm{mg}(* * *)$ \\
\hline $5 \mathbf{a}$ & 3,4-Dimethoxy phenyl & $\mathrm{H}$ & $2 \cdot 8 \pm 0 \cdot 1225$ & $2 \cdot 0 \pm 0 \cdot 1581$ \\
\hline $5 d$ & 2-Pyrazine & $\mathrm{H}$ & $2 \cdot 7 \pm 0 \cdot 1225$ & $2 \cdot 0 \pm 0 \cdot 1581$ \\
\hline $5 \mathbf{i}$ & 2-Pyrazine & $\mathrm{OCH}_{3}$ & $2 \cdot 4 \pm 0 \cdot 1871$ & $1 \cdot 8 \pm 0 \cdot 2$ \\
\hline $5 \mathbf{k}$ & 3,4-Dimethoxy phenyl & $\mathrm{OCHF}_{2}$ & $2 \cdot 2 \pm 0 \cdot 1225$ & $1 \cdot 5 \pm 0 \cdot 1581$ \\
\hline $5 \mathbf{l}$ & 2-Pyridyl & $\mathrm{OCHF}_{2}$ & $2 \cdot 6 \pm 0 \cdot 1871$ & $1 \cdot 6 \pm 0 \cdot 1$ \\
\hline $5 n$ & 2-Pyrazine & $\mathrm{OCHF}_{2}$ & $2 \cdot 4 \pm 0 \cdot 1$ & $1 \cdot 3 \pm 0 \cdot 1225$ \\
\hline 50 & 5-Tetrazole & $\mathrm{OCHF}_{2}$ & $2 \cdot 4 \pm 0 \cdot 1871$ & $1 \cdot 6 \pm 0.2915$ \\
\hline Omeprazole & & & $0 \cdot 3 \pm 0 \cdot 1225$ & $0 \cdot 0 \pm 0 \cdot 0$ \\
\hline Control & & & & $6 \cdot 0 \pm 0 \cdot 2236$ \\
\hline
\end{tabular}

$n=5$, Values are expressed as mean \pm SEM. $* P<0.1$ compared to control group (Student's $t$ test). $* * P<0.01$ compared to control group (Student's $t$ test). $* * * P<0.001$ compared to control group (Student's $t$ test).

The syntheses of the compounds were performed using the route shown in scheme 1. 2-(2-chloroacetamido)-N-substituted benzamide $\mathbf{2 a - e}$ were synthesized by condensation of 2-amino $N$-substituted benzamide 1 with chloroacetyl chloride by stirring for $8-10 \mathrm{~h}$ at $0^{\circ} \mathrm{C}$. The 2-chloromethyl-3-N-substituted quinazoline $4(3 \mathrm{H})$ one $(\mathrm{R}=3,4$ dimethoxy phenyl, 2-pyridyl, 3-pyridyl, 2-pyrazine, 5-tetrazole) 3a-e were synthesized by cyclisation of 2-(2chloroacetamido)-N-substituted benzamide refluxing with acetic anhydride for $10-12 \mathrm{~h}^{7,8}$ Compounds were confirmed by IR spectral data. Quinazoline ring showed band at $1668-1678 \mathrm{~cm}^{-1}$ indicating the presence of an $\mathrm{N}-\mathrm{CO}$ group. $\mathrm{NH}$ of benzamide $\left(\mathrm{NHCOCH}_{2} \mathrm{Cl}\right)$ showed band at 3190-3220.2-(2chloroacetamido)-N-substituted benzamide cyclized to form 2-chloromethyl compound showed band for
$\mathrm{C}=\mathrm{N}$ at $1605-1668, \mathrm{C}-\mathrm{N}$ at $1514-1550, \mathrm{C}-\mathrm{Cl}$ at 759-768, alkyl $\mathrm{C}-\mathrm{H}\left(\mathrm{CH}_{2} \mathrm{CI}\right)$ at 2930-2940.

2-chloromethyl-3-substituted quinazoline-4 $(3 \mathrm{H})$ ones 3a-e were then reacted with 2-mercapto benzimidazoles $\left(\mathrm{R}_{1}=\mathrm{H}, \mathrm{OCH}_{3}, \mathrm{OCHF}_{2}\right)$ by stirring for $10-15 \mathrm{~h}$ in $\mathrm{NaOH}$ solution by substitution reaction to give-2-[1- $H$-benzo(d)imidazol-2-yl thio]methyl3 -substituted quinazoline-4-(3H)ones. ${ }^{9}$ 4a-o. Mild oxidation of sulphur was carried out using $30 \% \mathrm{w} / \mathrm{v}$ of hydrogen peroxide in acetic acid in presence of dichloromethane and stirred for $3-5 \mathrm{~h}$ at $50-60^{\circ} \mathrm{C}$ to give targeted sulfinyl derivatives 5a-0. Chlorine atom of quinazoline, substituted by mercapto proton showed $\mathrm{C}-\mathrm{S}-\mathrm{C}$ peak in between 712 and 744 with disappearance of $\mathrm{C}-\mathrm{Cl}$ band. Oxidation of sulphur showed peak at 1021-1033 and shifts $\mathrm{C}-\mathrm{S}-\mathrm{C}$ peak to $716-785$. ${ }^{1} \mathrm{H}$ NMR spectra of compounds $\mathbf{5 a - 0}$ 
Table 2. Percentage of inhibition of gastric acid (5a-0).

\begin{tabular}{|c|c|c|c|c|c|}
\hline \multirow[b]{2}{*}{ Compound no. } & \multirow[b]{2}{*}{$R$} & \multicolumn{4}{|c|}{ Percentage of inhibition of acid secretion ( $20 \mathrm{mg}$ ) } \\
\hline & & $R_{1}$ & $\begin{array}{l}\text { Aspirin induced } \\
\text { ulcer }\end{array}$ & $\begin{array}{l}\text { Pylorus ligation- } \\
\text { induced ulcer }\end{array}$ & $\begin{array}{l}\text { Ethanol- } \\
\text { induced ulcer }\end{array}$ \\
\hline $5 \mathbf{a}$ & 3,4-Dimethoxy phenyl & $\mathrm{H}$ & 90 & 84 & 67 \\
\hline $5 \mathbf{b}$ & 2-Pyridyl & $\mathrm{H}$ & - & 83 & - \\
\hline $5 c$ & 3-Pyridyl & $\mathrm{H}$ & - & 79 & - \\
\hline $5 d$ & 2-Pyrazine & $\mathrm{H}$ & 92 & 84 & 67 \\
\hline $5 e$ & 5-Tetrazole & $\mathrm{H}$ & - & 76 & - \\
\hline $\mathbf{5 f}$ & 3,4-Dimethoxy phenyl & $\mathrm{OCH}_{3}$ & - & 84 & - \\
\hline $5 \mathrm{~g}$ & 2-Pyridyl & $\mathrm{OCH}_{3}$ & - & 83 & - \\
\hline $5 \mathbf{h}$ & 3-Pyridyl & $\mathrm{OCH}_{3}$ & - & 83 & - \\
\hline $5 \mathbf{i}$ & 2-Pyrazine & $\mathrm{OCH}_{3}$ & 95 & 90 & 70 \\
\hline $5 \mathbf{j}$ & 5-Tetrazole & $\mathrm{OCH}_{3}$ & - & 83 & - \\
\hline $5 \mathbf{k}$ & 3,4-Dimethoxy phenyl & $\mathrm{OCHF}_{2}$ & 95 & 92 & 75 \\
\hline $5 \mathbf{l}$ & 2-Pyridyl & $\mathrm{OCHF}_{2}$ & 87 & 88 & 73 \\
\hline $5 \mathrm{~m}$ & 3-Pyridyl & $\mathrm{OCHF}_{2}$ & - & 83 & - \\
\hline $5 n$ & 2-Pyrazine & $\mathrm{OCHF}_{2}$ & 97 & 92 & 78 \\
\hline 50 & 5-Tetrazole & $\mathrm{OCHF}_{2}$ & 97 & 88 & 73 \\
\hline Omeprazole & & & 100 & 100 & 100 \\
\hline
\end{tabular}

exhibited a singlet at $4 \cdot 8-5 \cdot 1$ and $11 \cdot 8-12 \cdot 4$ integrating for two and one protons respectively and assigned to methylene proton of the chain $\left(-\mathrm{CH}_{2}-\mathrm{S}-\right)$ and $\mathrm{N}-$ $\mathrm{H}$ of benzimidazole. A singlet for 6 protons at 3.8 3.9 is shown for $\mathrm{OCH}_{3}$ of 3,4 dimethoxy. Other signals appeared in the aromatic region ranging from 7.0 to 8.6 integrating for the protons of quinazoline, and benzimidazole nucleus.

\subsection{Antiulcer screening}

All the quinazoline-4(3H)-ones were evaluated for their antiulcer activity in pylorus ligation-induced ulcers in rats with ulcer index, $\mathrm{pH}$ and volume of gastric juice as shown in table la. Compounds which showed higher antiulcer and antisecretory activity were subjected to different screening methods such as aspirin and ethanol-induced ulcers in rats. Results are shown in tables $1 \mathrm{~b}$ and c. Compounds $\mathbf{5 k}$ and $\mathbf{5 n}$ showed most potent activity as compared to Omeprazole at the dose level of 10 and $20 \mathrm{mg} / \mathrm{kg}$. Compounds 5a, 5d, 5i, 5l and 5o showed moderate activity at the same doses. Compounds $\mathbf{5 i}, \mathbf{5 k}, \mathbf{5 n}$ at $20 \mathrm{mg} / \mathrm{kg}$ dose showed less ulcer index. Mostly compounds 5d, 5i, 5l, 5n and $\mathbf{5 o}$ at $10 \mathrm{mg} / \mathrm{kg}$ and compounds 5i, 5k, 5n, 5o and $\mathbf{5 l}$ at $20 \mathrm{mg} / \mathrm{kg}$ doses inhibited acid secretion and overall results are as shown in table 2 .

The perusal of the overall results have shown that benzimidazole sulfinyl methyl quinazoline substi- tuted with 3-N pyrazine, dimethoxy phenyl with difluromethoxy showed maximum activity. By observing the toxicity study, arbitrarily $10,20 \mathrm{mg} /$ $\mathrm{kg}$ doses of test compounds were selected. Further studies are in progress to optimize these lead compounds and to characterize the mode of action.

\subsection{Acute toxicity $\left(L D_{50}\right)$}

The synthesized compounds were tested in different doses in Albino mice as per guidelines (AOT No. 425) suggested by Organization for Economic Co-operation and Development (OECD) 2001 and did not produce any toxicity. The doses of the test compounds for antiulcer activity have been selected arbitrarily.

\section{Acknowledgement}

We are grateful to Science and Technology Park affiliated to University of Pune, for providing us the NMR and Mass Spectrometry facility and elemental analysis, for carrying out the research work.

\section{References}

1. Robert S and McDonald I M 2003 Burger's medicinal chemistry and drug discovery (New Jersey: John Wiley and Sons), 6th edn, pp 86-121 
2. Parnham I J and Bruinvels J 1999 Proton pump inhibitors (Swizarland: Birkhauser) p. 7

3. Hiroshi S, Koji T, Akito K, Yasuhiro I, Isami K, Asyoshi K, Mikiko K and Makota S 1995 Chem. Pharm. Bull. 43166

4. Haryulu P V R, Dubey P K, Prasad Reddy P V V and Thatipally S 2008 ARKIVOC VI 104

5. Ghabrial S and GaberHatem M 2003 Molecules 8 401
6. Vogel H G 2002 Drug discovery and evaluation (Berlin Heidelberg: Springer-Verlag) 2nd edn, p. 825

7. Kulkarni Y D, Abdi S H R and Sharma V L 1984 J. Indian Chem. Soc. LXI 720

8. Pattan S R, KrishanaReddy V V, Manvi F V, Desai B $\mathrm{G}$ and Bhat A R 2006 Indian J. Chem. B45 1778

9. Ife R J, Dyke C A, Keeling D J, Meemam E, Meesoon M L, Parson M E, Price C A, Theobald C J and Underwood A H 1989 J. Med. Chem. 321970 